\title{
AN EMPIRICAL STUDY OF TRANSBOUNDARY AIR POLLUTION OF THE BEIJING-TIANJIN REGION*
}

\author{
ZULIANG LU ${ }^{\dagger}$, FEI HUANG ${ }^{\dagger}$, LIN LI $^{\ddagger}$, XIAOXIAO ZUO ${ }^{\dagger}$, AND JUNHONG LI ${ }^{\dagger}$
}

\begin{abstract}
In this paper, we present a stochastic differential game to model the transboundary air pollution problems of the Beijing-Tianjin region with emission permits trading. By using stochastic optimal control theory, we obtain the Hamilton-Jacobi-Bellman equation satisfied by the value function for the cooperative games. Next, we solve the Hamilton-Jacobi-Bellman equation by using a fitted finite volume method. Finally, the efficiency and the usefulness of the fitted finite volume method are illustrated by an empirical study.
\end{abstract}

Key words. transboundary air pollution of the Beijing-Tianjin region, stochastic differential game, emission permits trading, Hamilton-Jacobi-Bellman equation, fitted finite volume method

AMS subject classifications. 49J20, 65N30

1. Introduction. Pollution is a severe environmental problem in the world. As an important part of environmental pollution, transboundary air pollution problems have extremely important effects on health and lives, and serious transboundary air pollution even constitutes great threats to the survival and the development of human beings, so it is inevitable to study transboundary air pollution problems.

Transboundary air pollution problems have attracted the attention of environmentalists, lawyers, economists, and political scientists; see, for instance, [1, 9, 17]. Transboundary air pollution in the Beijing-Tianjin region is an outstanding example of it in China and even around the world. The Beijing-Tianjin region is one of the important centers of economy and culture, and it has major influence. Solving the transboundary air pollution problems of the Beijing-Tianjin region has enormous significance. We focus on the transboundary industrial pollution problem in the Beijing-Tianjin region and discuss it within a differential game framework and employ the Hamilton-Jacobi-Bellman equation. By using the fitted finite volume method, we solve the Hamilton-Jacobi-Bellman equation and provide an empirical study.

The U.S. and Canada first completed a bargain deal on transboundary air pollution problems, i.e., when pollution originates at one location and damages another region's air quality after traveling. Differential games can be regarded as an effective instrument for dealing with pollution control problems and for examining the reciprocal actions between the dynamic processes of pollution and the participants' behaviors. They are often used to model and analyze the actions in the case of dynamic systems. In this approach, there are many players with their own goals in the system and the dynamics of the players' states are modeled by a series of differential equations. In a transboundary pollution control problem, the neighboring countries or regions can be seen as the players, and they aim at maximizing the joint or their own net present profits under a cooperative or non-cooperative games point of view, respectively.

* Received December 13, 2018. Accepted November 5, 2019. Published online on February 6, 2020. Recommended by Yves Achdou. This work is supported by National Science Foundation of China (11201510), National Social Science Fund of China (19BGL190), China Postdoctoral Science Foundation (2017T100155, 2015M580197), Innovation Team Building at Institutions of Higher Education in Chongqing (CXTDX201601035), and Chongqing Research Program of Basic Research and Frontier Technology (cstc2019jcyj-msxmX0280).

${ }^{\dagger}$ Key Laboratory for Nonlinear Science and System Structure, Chongqing Three Gorges University, Chongqing 404000, P.R.China; Research Center for Mathematics and Economics, Tianjin University of Finance and Economics, Tianjin, 300222, P.R.China ( $\{$ zulianglux, fei huangc, xiaoxzuo, junhonglio\} $@ 126 . c o m$ ).

${ }^{\ddagger}$ College of Computer Science and Technology, Chongqing University of Posts and Telecommunications, Chongqing, 400065, P.R.China (linligx@126.com). 


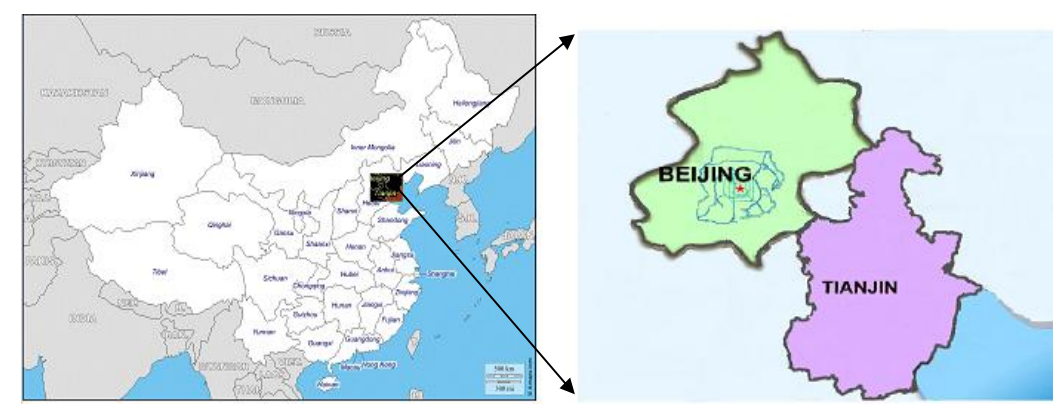
side.

FIG. 1.1. The map of China on the left-hand side and the map of Beijing city and Tianjing city on the right-hand

Recently, many researchers have paid attention to transboundary air pollution problems. For example, Yeung [15] first derived time-consistent solutions in a cooperative differential game and studied the pollution management in a stochastic differential game framework. In [16], the authors present a cooperative stochastic differential game of transboundary industrial pollution and derive a payment distribution mechanism, which maintains the subgame consistency. The authors study the impact of clean technology adoption with the finding that countries can cope with the increase of pollution by increasing emissions in the noncooperative transboundary pollution game [1]. Others have studied the harmonization of international and domestic law [9], abatement cost [14], and even R and D spillovers [17].

To our best knowledge, transboundary air pollution requires action by the international community to control its formation and impact. A cooperative differential game model of transboundary industrial pollution was presented by Yeung [15]. Then Li [12] extended Yeung's model to an even more general model, in which emission permits trading is taken into account. For our applications and the empirical study, we also employ this model. Moreover, Bernard [2] has tested the impact of the strategic interactions between Russia and China in the international carbon emission permits market. Zhang [6] generalized the emission permits price model obeying a geometric Brownian motion, which is commonly used to described the path of an underlying asset in financial markets. Our goal is to make use of optimal control theory to seek the cooperative optimal emission paths of the Beijing and Tianjing regions.

In this paper, we discuss a stochastic differential game to model the transboundary air pollution problems of the Beijing-Tianjin region with emission permits trading. We make use of stochastic optimal control theory to derive the Hamilton-Jacobi-Bellman equation satisfied by the value functions for the cooperative games. Furthermore, we solve the Hamilton-JacobiBellman equation by using a fitted finite volume method. The efficiency and the usefulness of this method are illustrated by an empirical study.

The outline of this paper is as follows. In Section 2, we provide the cooperative game formulations from which the Hamilton-Jacobi-Bellman equation is derived. Then, a fitted finite volume method is proposed for the discretization of the Hamilton-Jacobi-Bellman equation in Section 3. In Section 4, an empirical study is performed to illustrate the efficiency and usefulness of the numerical method.

2. The Hamilton-Jacobi-Bellman equation. In this section, we discuss the differential game framework and the Hamilton-Jacobi-Bellman equation for the transboundary air pollution problem of the Beijing-Tianjin region. In order to illustrate the dynamics of pollution and the 


\section{ETNA}

Kent State University and

Johann Radon Institute (RICAM)

interactions among the players in a commitment period, we propose a finite-horizon differential game framework. We assume that the game involves the Beijing-Tianjin region; it is a region that is not only a serious problem area of air pollution but also one of the pioneers in exploring transboundary treatment.

Let $Q_{i}(t)(i=1,2)$ denote the production of region $i$ (Beijing city and Tianjin city) during the period $[0, T]$, where $T$ is the maturity of the game. This production leads to an amount of by-products, namely emissions $E_{i}(t)$, which depend only on time $t$. Suppose that region $i$ 's net revenue arising from the production can be represented by an increasing concave function $R_{i}\left(Q_{i}(t)\right)$. Following [3, 4, 8, 12], we assume that the relationship between production and emissions is linear, and the production revenue function can be expressed by the following quadratic functional form in terms of the emissions:

$$
R_{i}\left(E_{i}(t)\right)=A_{i} E_{i}(t)-\frac{1}{2} E_{i}^{2}(t),
$$

where $A_{i}(i=1,2)$ are positive constants.

In an emission permits trading scheme, the initial quota $E_{i 0}$, which is a positive constant, is often allocated by the grandfather principle or auction principle. Then, the trading volume of the emission permits of region $i$ is given by

$$
Y_{i}(t)=E_{i}(t)-E_{i 0} .
$$

We assume that the emission permits price $S(t)$ is stochastic and follows a geometric Brownian motion:

$$
d S(t)=\mu_{S} S(t) d t+\sigma_{S} S(t) d W_{S},
$$

where $\mu_{S}$ and $\sigma_{S}$ are two constants representing the drift rate and the volatility of the emission permits price, respectively, and $d W_{S}$ denotes the increments to the standard Brownian process.

Region $i$ 's industrial net revenue involves emission permits trading with a stochastic dynamic price process at time $t$, and it can be written as

$$
\begin{aligned}
\Pi_{i}\left(E_{i}(t)\right) & =A_{i} E_{i}(t)-\frac{1}{2} E_{i}^{2}(t)-S(t)\left(E_{i}(t)-E_{i 0}\right) \\
& =\left(A_{i}-S(t)\right) E_{i}(t)-\frac{1}{2} E_{i}^{2}(t)+S(t) E_{i 0} .
\end{aligned}
$$

Moreover, let $P(t)$ denote the stock of pollution in the environment at time $t$. Then, the dynamic process of the pollution stock is governed by the following stochastic differential equation:

$$
d P(t)=\left(\left(\sum_{i=1}^{2} E_{i}(t)\right)-\theta_{P} P(t)\right) d t+\sigma_{P} P(t) d W_{P},
$$

where $E_{1}(t)$ and $E_{2}(t)$ denote the emission levels of regions 1 and 2, respectively. Here $\theta_{P}$ represents the exponential decay rate of the pollution. The constant $\sigma_{P}$ is a noise parameter and represents the volatility of the pollution stock, and $W_{P}$ is the increment to the standard Brownian process. In particular, we set $A_{2}=\alpha_{1} A_{1}$ and $D_{2}=\beta_{1} D_{1}$, where $D_{i}(i=1,2)$ is a strictly positive parameter. By means of [13], the parameters $\alpha$ and $\beta$ characterize the differences in the two regions' capacities in bearing damages from the stock of pollution or from abatement costs and in generating revenues from production.

Besides, we suppose that the salvage cost at time $T$ for the pollution stock is a linear function $g_{i}\left(\bar{P}_{i}-P(T)\right)$, where $g_{i}>0$ and $\bar{P}_{i}>0$. 
Hence, the current objective of region $i$ is to find an optimal plan which maximizes the expected value of the flow of the instantaneous net revenue. That is, the objective functional and constraint conditions of region $i$ are as follows:

$$
\begin{aligned}
\max _{E_{i}(t)} E\left\{\int_{0}^{T} \exp (-r t)\left[\left(A_{i}-S(t)\right) E_{i}(t)-\frac{1}{2} E_{i}^{2}(t)+S(t) E_{i 0}-D_{i} P(t)\right] d t\right\} \\
-g_{i}\left(P(T)-\bar{P}_{i}\right) \exp (-r T), \\
\text { subject to }\left\{\begin{array}{l}
d S(t)=\mu_{S} S(t) d t+\sigma_{S} S(t) d W_{S}, \\
S(0)=S_{0}, \\
d P(t)=\left(\left(\sum_{i=1}^{2} E_{i}(t)\right)-\theta_{P} P(t)\right) d t+\sigma_{P} P(t) d W_{P}, \\
P(0)=P_{0},
\end{array}\right.
\end{aligned}
$$

where $r$ is the social risk-free discount rate.

Suppose that the joint value function $V_{C}(P, S, t)$ is a twice continuously differentiable function of $P$ and $S$, and we assume that the two Brownian processes $W_{S}$ and $W_{P}$ are correlated with a correlation coefficient $\rho>0$; here $\rho=0$ means that they are independent of each other. By applying the dynamic programming approach and Ito's lemma to solve the above stochastic optimal control problem, we obtain the following Hamilton-Jacobi-Bellman equation satisfied by the value function $V_{C}(P, S, t)$ :

$$
\begin{aligned}
\max _{E_{C i}(t)} & \left\{\frac{\partial V_{C}}{\partial t}+\left(\left(\sum_{i=1}^{2} E_{C i}\right)-\theta_{P} P\right) \frac{\partial V_{C}}{\partial P}+\frac{1}{2} \sigma_{P}^{2} P^{2} \frac{\partial^{2} V_{C}}{\partial P^{2}}+\mu_{S} S \frac{\partial V_{C}}{\partial S}\right. \\
+ & \left.\frac{1}{2} \sigma_{S}^{2} S^{2} \frac{\partial^{2} V_{C}}{\partial S^{2}}+\rho \sigma_{P} \sigma_{S} P S \frac{\partial^{2} V_{C}}{\partial P \partial S}-r V_{C}+F_{C}\left(P, S, E_{C 1}, E_{C 2}, t\right)\right\}=0
\end{aligned}
$$

with the terminal condition

$$
V_{C}(P, S, T)=-\sum_{i=1}^{2} g_{i}\left(P(T)-\bar{P}_{i}\right)
$$

where

$$
F_{C}\left(P, S, E_{C 1}, E_{C 2}, t\right)=\sum_{i=1}^{2}\left(A_{i}-S\right) E_{C i}-\sum_{i=1}^{2} \frac{E_{C i}^{2}}{2}+\left(\sum_{i=1}^{2} E_{i 0}\right) S-\left(\sum_{i=1}^{2} D_{i}\right) P .
$$

3. Fitted finite volume method. It is easy to see that the Hamilton-Jacobi-Bellman equations (2.1)-(2.2) can not be solved analytically. In this section, we present a fitted finite volume method to discretize them. A two-level implicit time-stepping method is used to implement the time-discretization. 
Let us denote the optimal emission paths by $E_{C 1}^{*}$ and $E_{C 2}^{*}$. From the first-order optimality condition, we know that equation (2.1) can be split into the following coupled equations:

$$
\begin{aligned}
& \frac{\partial V_{C}}{\partial t}+\left(E_{C 1}^{*}(t)+E_{C 2}^{*}(t)-\theta_{P} P\right) \frac{\partial V_{C}}{\partial P}+\frac{1}{2} \sigma_{P}^{2} P^{2} \frac{\partial^{2} V_{C}}{\partial P^{2}}+\mu_{S} S \frac{\partial V_{C}}{\partial S}+\frac{1}{2} \sigma_{S}^{2} S^{2} \frac{\partial^{2} V_{C}}{\partial S^{2}} \\
&+\rho \sigma_{P} \sigma_{S} P S \frac{\partial^{2} V_{C}}{\partial P \partial S}-r V_{C}+F_{C}\left(P, S, E_{C 1}^{*}(t), E_{C 2}^{*}(t), t\right)=0, \\
& E_{C 1}^{*}(P, S, t)=A_{1}-S+\frac{\partial V_{C}}{\partial P}, \\
& E_{C 2}^{*}(P, S, t)=A_{2}-S+\frac{\partial V_{C}}{\partial P} .
\end{aligned}
$$

Now, we introduce the fitted finite volume method for the spatial discretization. Essential for the discretization is a mesh for $\left(P_{\min }, P_{\max }\right) \times\left(S_{\min }, S_{\max }\right)$. We first divide the intervals $I_{P}$ and $I_{S}$ into $N_{P}$ and $N_{S}$ subintervals, respectively:

$$
I_{P_{i}}:=\left(P_{i}, P_{i+1}\right), \quad I_{S_{j}}:=\left(S_{j}, S_{j+1}\right), \quad i=0,1, \ldots, N_{P}-1, \quad j=0,1, \ldots, N_{S}-1,
$$

where

$$
P_{\min }=P_{0}<P_{1}<\cdots<P_{N_{P}}=P_{\max } \quad \text { and } \quad S_{\min }=S_{0}<S_{1}<\cdots<S_{N_{S}}=S_{\max } .
$$

Thus, a mesh on $I_{P} \times I_{S}$ is defined whose mesh lines are perpendicular to the axes. Next we define another partition of $I_{P} \times I_{S}$ by letting

$$
P_{i-\frac{1}{2}}=\frac{P_{i-1}+P_{i}}{2}, P_{i+\frac{1}{2}}=\frac{P_{i}+P_{i+1}}{2}, S_{j-\frac{1}{2}}=\frac{S_{j-1}+S_{j}}{2}, S_{j+\frac{1}{2}}=\frac{S_{j}+S_{j+1}}{2},
$$

for any $i=1,2, \ldots, N_{P}-1$ and $j=1,2, \ldots, N_{S}-1$. For completeness, we also set

$$
P_{-\frac{1}{2}}=P_{\min }, P_{N_{P}+\frac{1}{2}}=P_{\max }, S_{-\frac{1}{2}}=S_{\min } S_{N_{S}+\frac{1}{2}}=S_{\max } .
$$

The stepsizes are given by $h_{P_{i}}=P_{i+\frac{1}{2}}-P_{i-\frac{1}{2}}$ and $h_{S_{j}}=S_{j+\frac{1}{2}}-S_{j-\frac{1}{2}}$ for each $i=0,1, \ldots, N_{P}$ and $j=0,1, \ldots, N_{S}$.

Then, for the purpose of formulating a finite volume scheme [7, 11], we write equation (3.1a) in the following divergence form:

$$
-\frac{\partial V_{C}}{\partial t}-\nabla \cdot\left(A \nabla V_{C}+\underline{b} V_{C}\right)+c V_{C}=F_{C},
$$

where

$$
\begin{aligned}
A & =\left[\begin{array}{ll}
a_{11} & a_{12} \\
a_{21} & a_{22}
\end{array}\right]=\left[\begin{array}{cc}
\frac{1}{2} \sigma_{P}^{2} P^{2} & \frac{1}{2} \rho \sigma_{P} \sigma_{S} P S \\
\frac{1}{2} \rho \sigma_{P} \sigma_{S} P S & \frac{1}{2} \sigma_{S}^{2} S^{2}
\end{array}\right], \\
\underline{b} & =\left[\begin{array}{l}
b_{1} \\
b_{2}
\end{array}\right]=\left[\begin{array}{c}
E_{C 1}^{*}+E_{C 2}^{*}-\theta_{P} P-\sigma_{P}^{2} P-\frac{1}{2} \rho \sigma_{P} \sigma_{S} P \\
\mu_{S} S-\sigma_{S}^{2} S-\frac{1}{2} \rho \sigma_{P} \sigma_{S} S
\end{array}\right], \\
c & =r+\mu_{S}+2 \frac{\partial^{2} V_{C}}{\partial P^{2}}-\theta_{P}-\sigma_{P}^{2}-\sigma_{S}^{2}-\rho \sigma_{P} \sigma_{S} .
\end{aligned}
$$

It follows by integrating equation (3.2) over $\mathcal{R}_{i, j}=\left[S_{i-\frac{1}{2}}, S_{i+\frac{1}{2}}\right] \times\left[\delta_{j-\frac{1}{2}}, \delta_{j+\frac{1}{2}}\right]$ and applying the midpoint quadrature rule to the resulting equation that

$$
-\frac{\partial V_{C_{i, j}}}{\partial t} R_{i, j}-\iint_{\mathcal{R}_{i, j}} \nabla \cdot\left(A \nabla V_{C}+\underline{b} V_{C}\right) d P d S+c_{i, j} V_{C_{i, j}} R_{i, j}=F_{C_{i, j}} R_{i, j},
$$


for $i=1,2, \ldots, N_{P}-1, j=1,2, \ldots, N_{S}-1$, where $R_{i, j}=\left(P_{i+\frac{1}{2}}-P_{i-\frac{1}{2}}\right) \times\left(S_{j+\frac{1}{2}}-S_{j-\frac{1}{2}}\right)$, $c_{i, j}=c\left(P_{i}, S_{j}, t\right), V_{C_{i, j}}=V_{C}\left(P_{i}, S_{j}, t\right)$, and $F_{C_{i, j}}=F_{C}\left(P_{i}, S_{j}, E_{C 1}^{*}, E_{C 2}^{*}, t\right)$.

The approximation of the second term in equation (3.3) is the difficult key point of this numerical method. According to the definition of the flux $A \nabla V_{C}+\underline{b} V_{C}$ and by integrating by parts, we have

$$
\begin{aligned}
\iint_{\mathcal{R}_{i, j}} & \nabla \cdot\left(A \nabla V_{C}+\underline{b} V_{C}\right) d S d \delta \\
= & \int_{\partial \mathcal{R}_{i, j}}\left(A \nabla V_{C}+\underline{b} V_{C}\right) \cdot l d s \\
= & \int_{\left(P_{i+\frac{1}{2}}, S_{j-\frac{1}{2}}\right)}^{\left(P_{i+\frac{1}{2}}, S_{j+\frac{1}{2}}\right)}\left(a_{11} \frac{\partial V_{C}}{\partial P}+a_{12} \frac{\partial V_{C}}{\partial S}+b_{1} V_{C}\right) d S \\
& -\int_{\left(P_{i-\frac{1}{2}}, S_{j-\frac{1}{2}}\right)}^{\left(P_{i-\frac{1}{2}}, S_{j+\frac{1}{2}}\right)}\left(a_{11} \frac{\partial V_{C}}{\partial P}+a_{12} \frac{\partial V_{C}}{\partial S}+b_{1} V_{C}\right) d S \\
& +\int_{\left(P_{i-\frac{1}{2}}, S_{j+\frac{1}{2}}\right)}^{\left(P_{i+\frac{1}{2}}, S_{j+\frac{1}{2}}\right)}\left(a_{21} \frac{\partial V_{C}}{\partial P}+a_{22} \frac{\partial V_{C}}{\partial S}+b_{2} V_{C}\right) d P \\
& -\int_{\left(P_{i+\frac{1}{2}}, S_{j-\frac{1}{2}}\right)}^{\left(P_{i-\frac{1}{2}}, S_{j-\frac{1}{2}}\right)}\left(a_{21} \frac{\partial V_{C}}{\partial P}+a_{22} \frac{\partial V_{C}}{\partial S}+b_{2} V_{C}\right) d P
\end{aligned}
$$

where $l$ denotes the unit vector outward-normal to $\partial \mathcal{R}_{i, j}$. We approximate the first integral of equation (3.4) by a constant:

$$
\begin{aligned}
& \int_{\left(P_{i+\frac{1}{2}}, S_{j-\frac{1}{2}}\right)}^{\left(P_{i+\frac{1}{2}}, S_{j+\frac{1}{2}}\right)}\left(a_{11} \frac{\partial V_{C}}{\partial P}+a_{12} \frac{\partial V_{C}}{\partial S}+b_{1} V_{C}\right) d S \\
& \left.\quad \approx\left(a_{11} \frac{\partial V_{C}}{\partial P}+a_{12} \frac{\partial V_{C}}{\partial S}+b_{1} V_{C}\right)\right|_{\left(P_{i+\frac{1}{2}}, S_{j}\right)} \cdot h_{S_{j}} .
\end{aligned}
$$

Now, we are in the position to derive the approximations to $\left(a_{11} \frac{\partial V_{C}}{\partial P}+a_{12} \frac{\partial V_{C}}{\partial S}+b_{1} V_{C}\right)$ at the midpoint $\left(P_{i+\frac{1}{2}}, S_{j}\right)$ of the interval $I_{P_{i}}$ for any $i=0,1, \ldots, N_{P}-1$. To begin with, the term $a_{11} \frac{\partial V_{C}}{\partial P}+b_{1} V_{C}$ is approximated by a constant, which means that its derivative equals zero, that is,

$$
\begin{gathered}
\left(\frac{1}{2} \sigma_{P}^{2} P^{2} \frac{\partial V_{C}}{\partial P}+\left(E_{C 1}^{*}+E_{C 2}^{*}+E_{C 3}^{*}-\theta_{P} P-\sigma_{P}^{2} P-\frac{1}{2} \rho \sigma_{P} \sigma_{S} P\right) V_{C}\right)^{\prime} \\
\equiv\left(a P^{2} \frac{\partial V_{C}}{\partial P}+b_{1}^{i+\frac{1}{2}, j} V_{C}\right)^{\prime}=0 \\
V_{C}\left(P_{i}, S_{j}\right)=V_{C_{i, j}} \\
V_{C}\left(P_{i+1}, S_{j}\right)=V_{C_{i+1, j}}
\end{gathered}
$$

where $a=\frac{1}{2} \sigma_{P}^{2}, b_{1}^{i+\frac{1}{2}, j}=b_{1}\left(P_{i+\frac{1}{2}}, S_{j}\right)$, and $V_{C_{i, j}}$ and $V_{C_{i+1, j}}$ denote the values of $V_{C}$ at $\left(P_{i}, S_{j}\right)$ and $\left(P_{i+1}, C_{j}\right)$, respectively. A first-order ordinary differential equation is obtained by integrating both sides of equation (3.5a):

$$
a P^{2} \frac{\partial V_{C}}{\partial P}+b_{1}^{i+\frac{1}{2}, j} V_{C}=C_{1}
$$


where $C_{1}$ is an arbitrary constant that can be determined by the boundary conditions (3.5b) as follows [18]:

$$
C_{1}=b_{1}^{i+\frac{1}{2}, j} \frac{V_{C_{i+1, j}} \exp \left(-\frac{\alpha_{i, j}}{P_{i+1}}\right)-V_{C_{i, j}} \exp \left(-\frac{\alpha_{i, j}}{P_{i}}\right)}{\exp \left(-\frac{\alpha_{i, j}}{P_{i+1}}\right)-\exp \left(-\frac{\alpha_{i, j}}{P_{i}}\right)},
$$

where $\alpha_{i, j}=b_{1}^{i+\frac{1}{2}, j} / a$. Additionally, the derivative $\partial V_{C} / \partial S$ can be approximated by a forward difference $\left(V_{C_{i, j+1}}-V_{C_{i, j}}\right) / h_{S_{j}}$. As a result, we have

$$
\begin{aligned}
& \left.\left(a_{11} \frac{\partial V_{C}}{\partial P}+a_{12} \frac{\partial V_{C}}{\partial S}+b_{1} V_{C}\right)\right|_{\left(P_{i+\frac{1}{2}}, S_{j}\right)} \cdot h_{S_{j}} \\
\approx & \left(b_{1}^{i+\frac{1}{2}, j} \frac{V_{C_{i+1, j}} \exp \left(-\frac{\alpha_{i, j}}{P_{i+1}}\right)-V_{C_{i, j}} \exp \left(-\frac{\alpha_{i, j}}{P_{i}}\right)}{\exp \left(-\frac{\alpha_{i, j}}{P_{i+1}}\right)-\exp \left(-\frac{\alpha_{i, j}}{P_{i}}\right)}+d_{i, j} \frac{V_{C_{i, j+1}}-V_{C_{i, j}}}{h_{S_{j}}}\right) \cdot h_{S_{j}},
\end{aligned}
$$

where $d=\frac{1}{2} \rho \sigma_{P} \sigma_{S} P S$ and $d_{i, j}=d\left(P_{i}, S_{j}\right)$. Applying a similar method to the other three terms in equation (3.4), we get the following results:

$$
\begin{gathered}
\left.\left(a_{11} \frac{\partial V_{C}}{\partial P}+a_{12} \frac{\partial V_{C}}{\partial S}+b_{1} V_{C}\right)\right|_{\left(P_{i-\frac{1}{2}}, S_{j}\right)} \cdot h_{S_{j}} \\
\approx\left(b_{1}^{i-\frac{1}{2}, j} \frac{V_{C_{i, j}} \exp \left(-\frac{\alpha_{i-1, j}}{P_{i}}\right)-V_{C_{i-1, j}} \exp \left(-\frac{\alpha_{i-1, j}}{P_{i-1}}\right)}{\exp \left(-\frac{\alpha_{i-1, j}}{P_{i}}\right)-\exp \left(-\frac{\alpha_{i-1, j}}{P_{i-1}}\right)}+d_{i, j} \frac{V_{C_{i, j+1}}-V_{C_{i, j}}}{h_{S_{j}}}\right) \cdot h_{S_{j}}, \\
\left.\left(a_{21} \frac{\partial V_{C}}{\partial P}+a_{22} \frac{\partial V_{C}}{\partial S}+b_{2} V_{C}\right)\right|_{\left(P_{i}, S_{j+\frac{1}{2}}\right.} \cdot h_{P_{i}} \\
\approx S_{j+\frac{1}{2}}\left(\bar{b}_{i, j+\frac{1}{2}} \frac{S_{j+1}^{\bar{\alpha}_{i, j}} V_{C_{i+1, j}}-S_{j}^{\bar{\alpha}_{i, j}} V_{C_{i, j}}}{S_{j+1}^{\bar{\alpha}_{i, j}}-S_{j}^{\bar{\alpha}_{i, j}}}+\bar{d}_{i, j} \frac{V_{C_{i, j+1}}-V_{C_{i, j}}}{h_{P_{i}}}\right) \cdot h_{P_{i}},
\end{gathered}
$$

and

$$
\begin{aligned}
& \left.\left(a_{21} \frac{\partial V_{C}}{\partial P}+a_{22} \frac{\partial V_{C}}{\partial S}+b_{2} V_{C}\right)\right|_{\left(P_{i}, S_{j-\frac{1}{2}}\right)} \cdot h_{P_{i}} \\
\approx & S_{j-\frac{1}{2}}\left(\bar{b}_{i, j-\frac{1}{2}} \frac{S_{j}^{\bar{\alpha}_{i, j-1}} V_{C_{i, j}}-S_{j-1}^{\bar{\alpha}_{i, j-1}} V_{C_{i, j-1}}}{S_{j}^{\bar{\alpha}_{i, j-1}}-S_{j-1}^{\bar{\alpha}_{i, j-1}}}+\bar{d}_{i, j} \frac{V_{C_{i, j+1}}-V_{C_{i, j}}}{h_{P_{i}}}\right) \cdot h_{P_{i}},
\end{aligned}
$$

where $\bar{\alpha}_{i, j}=\bar{b}_{i, j+\frac{1}{2}} / \bar{a}_{j}, \bar{a}=\frac{1}{2} \sigma_{S}^{2}, \bar{b}=\mu-\sigma_{S}^{2}-\frac{1}{2} \rho \sigma_{P} \sigma_{S}$, and $\bar{d}_{i, j}=\frac{1}{2} \rho \sigma_{P} \sigma_{S} P_{i}$. Hence, we obtain the following equations by combining equations (3.3), (3.4), and (3.6)-(3.9):

$$
\begin{aligned}
-\frac{\partial V_{C_{i, j}}}{\partial t} R_{i, j}+e_{i-1, j}^{i, j} V_{C_{i-1, j}}+e_{i, j-1}^{i, j} V_{C_{i, j-1}}+e_{i, j}^{i, j} V_{C_{i, j}}+e_{i, j+1}^{i, j} V_{C_{i, j+1}} & +e_{i+1, j}^{i, j} V_{C_{i+1, j}} \\
& =F_{C_{i, j}} R_{i, j}
\end{aligned}
$$


where

$$
\begin{aligned}
e_{i-1, j}^{i, j} & =-b_{1}^{i-\frac{1}{2}, j} \frac{\exp \left(-\frac{\alpha_{i-1, j}}{P_{i-1}}\right) h_{S_{j}}}{\exp \left(-\frac{\alpha_{i-1, j}}{P_{i}}\right)-\exp \left(-\frac{\alpha_{i-1, j}}{P_{i-1}}\right)}, \\
e_{i, j-1}^{i, j} & =-S_{j-\frac{1}{2}} \bar{b}_{i, j-\frac{1}{2}} \frac{S_{j-1}^{\bar{\alpha}_{i, j}-1} h_{P_{i}}}{S_{j}^{\bar{\alpha}_{i, j-1}}-S_{j-1}^{\bar{\alpha}_{i, j-1}}}, \\
e_{i, j}^{i, j} & =h_{S_{j}}\left(\frac{b_{1}^{i+\frac{1}{2}, j} \exp \left(-\frac{\alpha_{i, j}}{P_{i}}\right)}{\exp \left(-\frac{\alpha_{i, j}}{P_{i+1}}\right)-\exp \left(-\frac{\alpha_{i, j}}{P_{i}}\right)}+\frac{b_{1}^{i-\frac{1}{2}, j} \exp \left(-\frac{\alpha_{i-1, j}}{P_{i}}\right)}{\exp \left(-\frac{\alpha_{i-1, j}}{P_{i}}\right)-\exp \left(-\frac{\alpha_{i-1, j}}{P_{i-1}}\right)}+\bar{d}_{i, j}\right) \\
& +h_{P_{i}}\left(S_{j+\frac{1}{2}} \frac{\bar{b}_{i, j+\frac{1}{2}} S_{j}^{\bar{\alpha}_{i, j}}}{\left.S_{j+1}^{\bar{\alpha}_{i, j}}-S_{j}^{\bar{\alpha}_{i, j}}+S_{j-\frac{1}{2}} \frac{\bar{b}_{i, j-\frac{1}{2}}}{S_{j}^{\alpha_{i, j-1}}-S_{j-j}^{\bar{\alpha}_{i, j}}}\right)+c_{i, j}^{\bar{\alpha}_{i, j-1}} R_{i, j},}\right. \\
e_{i, j+1}^{i, j} & =-S_{j+\frac{1}{2}} \bar{b}_{i, j+\frac{1}{2}} \frac{S_{j+1}^{\bar{\alpha}_{i, j}} h_{P_{i}}}{S_{j+1}^{\bar{\alpha}_{i, j}}-S_{j}^{\bar{\alpha}_{i, j}}}, \\
e_{i+1, j}^{i, j} & =-b_{1}^{i+\frac{1}{2}, j} \frac{\exp \left(-\frac{\alpha_{i, j}}{P_{i+1}}\right) h_{S_{j}}}{\exp \left(-\frac{\alpha_{i, j}}{P_{i+1}}\right)-\exp \left(-\frac{\alpha_{i, j}}{P_{i}}\right)}-h_{S_{j}} \bar{d}_{i, j},
\end{aligned}
$$

for $i=1, \ldots, N_{P}-1, j=1, \ldots, N_{S}-1$. The other elements $e_{m, n}^{i, j}$ equal zero if $m \neq i-1, i, i+1$ and $n \neq j-1, j, j+1$. We can observe that (3.10) represents an $\left(N_{P}-1\right)^{2} \times\left(N_{S}-1\right)^{2}$ linear system of equations for

$$
V_{C}=\left(V_{C_{1,1}}, \ldots, V_{C_{1, N_{S}-1}}, V_{C_{2,1}}, \ldots, V_{C_{2, N_{S}-1}}, \ldots, V_{C_{N_{P}-1,1}}, \ldots, V_{C_{N_{P}-1, N_{S}-1}}\right)^{T} .
$$

Note that $V_{C_{0, j}}(t), V_{C_{i, 0}}(t), V_{C_{0, N_{S}}}(t)$, and $V_{C_{N_{P}, 0}}(t)$, for $i=1, \ldots, N_{P}$ and $j=1, \ldots, N_{S}$, equal the given boundary conditions. Obviously, the coefficient matrix of the system (3.10) is pentadiagonal.

Next we discuss the time-discretization of the system (3.10). For this purpose, we first rewrite equation (3.10) as

$$
-\frac{\partial V_{C_{i, j}}}{\partial t} R_{i, j}+D_{i, j} V_{C}=F_{C_{i, j}} R_{i, j}
$$

where

$$
D_{i, j}=\left(0, \ldots, 0, e_{i-1, j}^{i, j}, 0, \ldots, 0, e_{i, j-1}^{i, j}, e_{i, j}^{i, j}, e_{i, j+1}^{i, j}, 0, \ldots, 0, e_{i+1, j}^{i, j}, 0, \ldots, 0\right)
$$

for $i=1, \ldots, N_{P}-1$ and $j=1, \ldots, N_{S}-1$. We select $M-1$ points in $(0, T)$ numbered from $t_{1}$ to $t_{M-1}$, and let $T=t_{0}, t_{M}=0$, to form a partition of time $T=t_{0}>t_{1}>\cdots>t_{M}=0$. Then, the fully discrete form of equation (3.11) is obtained by applying the two-level implicit time-stepping method with a splitting parameter $\theta \in\left[\frac{1}{2}, 1\right]$ :

$$
\begin{aligned}
(\theta D(P, S, & \left.\left.E_{C 1}^{*}\left(t^{m+1}\right), E_{C 2}^{*}\left(t^{m+1}\right), E_{C 3}^{*}\left(t^{m+1}\right), t^{m+1}\right)+G^{m}\right) V_{C}^{m+1} \\
= & \theta F_{C}\left(P, S, E_{C 1}^{*}\left(t^{m+1}\right), E_{C 2}^{*}\left(t^{m+1}\right), E_{C 3}^{*}\left(t^{m+1}\right), t^{m+1}\right) \\
& +(1-\theta) F_{C}\left(P, S, E_{C 1}^{*}\left(t^{m}\right), E_{C 2}^{*}\left(t^{m}\right), E_{C 3}^{*}\left(t^{m}\right), t^{m}\right) \\
& +\left(G^{m}-(1-\theta) D\left(P, S, E_{C 1}^{*}\left(t^{m}\right), E_{C 2}^{*}\left(t^{m}\right), E_{C 3}^{*}\left(t^{m}\right), t^{m}\right)\right) V_{C}^{m},
\end{aligned}
$$


where

$$
\begin{aligned}
V_{C}^{m} & =\left(V_{C_{1,1}}^{m}, \ldots, V_{C_{1, N_{S}-1}}^{m}, V_{C_{2,1}}^{m}, \ldots, V_{C_{2, N_{S}}-1}^{m}, \ldots, V_{C_{N_{P}-1,1}}^{m}, \ldots, V_{C_{N-1, N_{S}}-1}^{m}\right)^{T}, \\
G^{m} & =\operatorname{diag}\left(-R_{1,1} / \Delta t_{m}, \ldots,-R_{N_{P}-1, N_{S}-1} / \Delta t_{m}\right)^{T},
\end{aligned}
$$

for $m=0,1, \ldots, M-1$. Note that $\Delta t_{m}=t_{m+1}-t_{m}<0$ and $V_{C}^{m}$ denotes the approximation of $V_{C}$ at $t=t_{m}$. Particularly, when we set $\theta=\frac{1}{2}$, the scheme (3.12) becomes the famous second-order accurate Crank-Nicolson scheme; if we set $\theta=1$, the scheme (3.12) becomes the first-order accurate backward Euler scheme. According to [5, Theorem 4], the system matrix of (3.12) is an M-matrix.

4. An empirical study. In this section, we perform an empirical study for transboundary air pollution of the Beijing-Tianjin region. Firstly, we give some economic data of Beijing city and Tianjing city in Table 4.1. GDP denotes the gross domestic product (hundred million yuan), and mean denotes the quadratic mean, $\sqrt{\left(a_{1}^{2}+\cdots+a_{n}^{2}\right) / n}$, for $n \in R$ and $a_{i}\left(a_{i}\right.$ denotes GDP or growth rates).

TABLE 4.1

The gross domestic product and economic growth rate of Beijing city and Tianjing city.

\begin{tabular}{ccccc} 
year & \multicolumn{2}{c}{ Beijing city } & \multicolumn{2}{c}{ Tianjing city } \\
& GDP & growth rates & GDP & growth rates \\
\hline 2006 & 8118 & 12.0 & 4463 & 13.1 \\
2007 & 9847 & 13.1 & 5253 & 15.5 \\
2008 & 11115 & 9.1 & 6719 & 16.5 \\
2009 & 12153 & 10.2 & 7522 & 16.5 \\
2010 & 14114 & 10.3 & 9224 & 17.4 \\
2011 & 16252 & 8.1 & 11307 & 16.4 \\
2012 & 17879 & 7.7 & 12894 & 13.8 \\
2013 & 19801 & 7.7 & 14442 & 12.5 \\
2014 & 21331 & 7.3 & 15727 & 10.0 \\
2015 & 23015 & 6.9 & 16538 & 9.3 \\
\hline mean & 15363 & 9.4 & 10409 & 13.1
\end{tabular}

According to the derivation of the air transboundary pollution model and references [12, $14,15]$, the parameters are chosen as $\theta_{P}=0.06, \beta=1.2, \rho=0.5$, and $r=0.08$. In compliance with other areas and considering reference [6], the other parameters are chosen to be $A_{1}=5, D_{1}=0.1, \sigma_{S}=0.3, \mu_{S}=0.2, g_{i}=3, g_{j}=2, \bar{P}_{i}=1100$, and $\bar{P}_{j}=1200$. For the empirical analysis, we set $T=10, E_{i 0}=5, E_{j 0}=6, P_{\max }=2000, S_{\min }=20$, $S_{\max }=50$, and $\sigma_{P}=0.3$, which are reasonable values and come from certain authoritative bodies $^{1}$.

In this example, the initial quotas $E_{10}$ and $E_{20}$ are both set to be very large, and the emission levels do not exceed them, so the two regions can sell their unused emission permits, and the net revenues $V_{C i}$ and $V_{N i}$ will increase with the increasing permits price $S$. Besides, the first-order conditions show that the optimal emission levels of the two regions can be expressed as

$$
E_{C i}^{*}=A_{i}-S+\frac{\partial V_{C i}}{\partial P},
$$

\footnotetext{
${ }^{1}$ http: //data.stats.gov.cn
} 


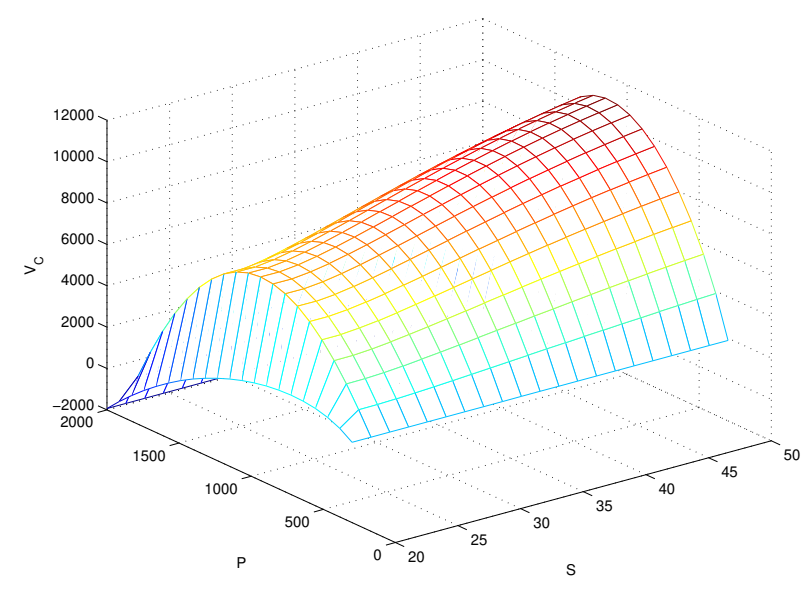

FIG. 4.1. The value function based on pollution stock and permits price.

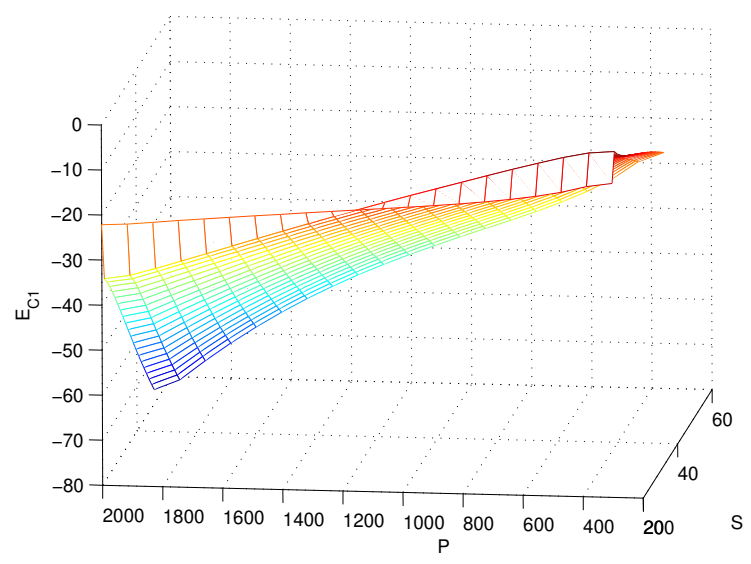

FIG. 4.2. The emission level of region 1 based on pollution stock and permits price.

for $i=1,2$. From the above equations, we can clearly see that the emission levels should decrease monotonically with increasing permit prices. This implies that the existence of the emission permit trading scheme does influence the players' decisions in the games.

Now, we illustrate the results by presenting some figures and tables. In Figure 4.1, the value function based on the pollution stock and the permits price is presented. Figure 4.1 demonstrates that the value function is higher when the state pollution stock is fixed and the emission permits prices are large. When the pollution stock is fixed, if the players intend to increase the value function of the two regions (Beijing city and Tianjing city), then they should increase the permits price.

Next, the emission levels of regions 2 and 3 based on th pollution and the price are presented in Figures 4.2 and 4.3. Here red parts represent higher emission levels and blue parts lower ones. It is clear that the trends of the emission level for both the two regions (Beijing city and Tianjing city) are consistent. It is also clear that the value function will be reduced when the pollution stock increases. 


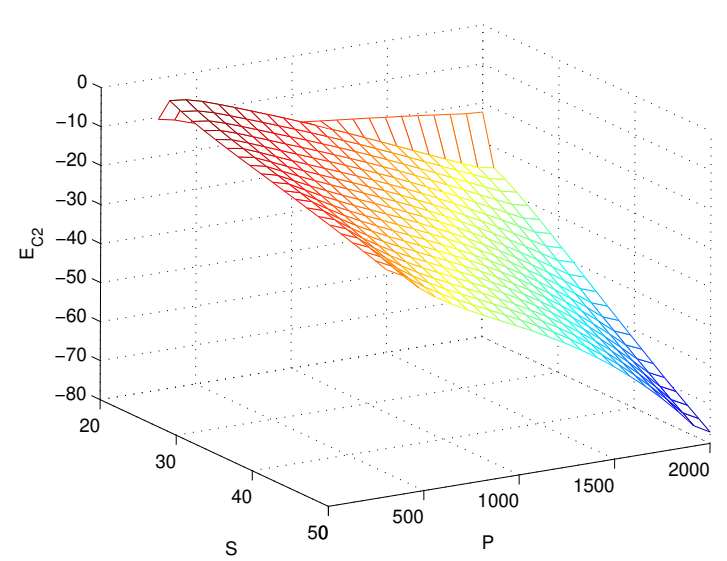

FIG. 4.3. The emission level of region 2 based on pollution stock and permits price.

TABle 4.2

The cooperative game at $t=0$.

\begin{tabular}{ccccccc}
$\begin{array}{c}\text { pollution } \\
\text { stock }\end{array}$ & permits & price & value function & \multicolumn{2}{c}{ emission levels } & \multicolumn{2}{c}{ trading volumes } \\
$P$ & $S$ & $V_{C}$ & $E_{C 1}$ & $E_{C 2}$ & $Y_{C 1}$ & $Y_{C 2}$ \\
\hline 470 & 23 & 2938.1 & -3.16 & -3.19 & -8.16 & -9.19 \\
& 29 & 6110.4 & -10.18 & -10.43 & -15.18 & -16.43 \\
& 35 & 7179.9 & -16.61 & -16.86 & -21.61 & -22.86 \\
\hline 810 & 23 & 3065.7 & -9.33 & -9.58 & -14.33 & -15.58 \\
& 29 & 6467.7 & -15.78 & -16.03 & -20.78 & -22.03 \\
& 35 & 7642.4 & -22.37 & -22.62 & -27.37 & -28.62 \\
\hline 1150 & 23 & 3293.2 & -12.96 & -13.21 & -17.96 & -19.21 \\
& 29 & 7207.1 & -20.79 & -21.04 & -25.79 & -27.04 \\
& 35 & 8626.3 & -27.90 & -28.15 & -32.90 & -34.15
\end{tabular}

In Tables 4.2 and 4.3, the value functions, emission levels, and trading volumes for the pollution stock and the permit prices at time $t=0$ and $t=5$ are presented. The trading volumes $Y$ for each table are computed by using the equations $Y_{i}(t)=E_{i}(t)-E_{i 0}$, for $i=1,2$. We can see from Tables 4.2 and 4.3 that the value function is higher when the permits price and the pollution stock increases for regions 1 and 2 .

Figure 4.4 depicts the value functions at $t=0$ and $t=5$. Comparing with the numerical results, it demonstrates that our numerical method for the Hamilton-Jacobi-Bellman equation governing the stochastic differential game in the transboundary air pollution problems of the Beijing-Tianjin region is useful. Thus, we have reason to believe that the fitted finite volume method can be a good tool for modeling the cooperative stochastic differential game for the transboundary air pollution problems of the Beijing-Tianjin region, so it can motivate the players to make more flexible decisions in the game. That is, with the increase of the emission price, the value will increase, while there will be a certain reduction when the price increases to a certain point so that the volume of emissions trading in the Beijing-Tianjin region will decrease. 
TABLE 4.3

The cooperative game at $t=5$.

\begin{tabular}{ccccccc}
$\begin{array}{c}\text { pollution } \\
\text { stock }\end{array}$ & permits & price & value function & \multicolumn{2}{c}{ emission levels } & \multicolumn{2}{c}{ trading volumes } \\
$P$ & $S$ & $V_{C}$ & $E_{C 1}$ & $E_{C 2}$ & $Y_{C 1}$ & $Y_{C 2}$ \\
\hline 470 & 23 & 3697.0 & -6.91 & -7.16 & -11.91 & -13.16 \\
& 29 & 5787.3 & -13.66 & -13.91 & -18.66 & -19.91 \\
& 35 & 5836.5 & -18.84 & -19.09 & -23.84 & -25.09 \\
\hline 810 & 23 & 3850.8 & -11.34 & -11.59 & -16.34 & -17.59 \\
& 29 & 6262.7 & -19.07 & -19.32 & -24.07 & -25.32 \\
& 35 & 6445.9 & -24.61 & -23.20 & -29.61 & -29.20 \\
\hline 810 & 23 & 3850.8 & -11.34 & -11.59 & -16.34 & -17.59 \\
1150 & 23 & 4103.1 & -14.68 & -13.20 & -19.68 & -19.20 \\
& 29 & 7103.1 & -23.97 & -23.12 & -28.97 & -29.12 \\
& 35 & 7531.7 & -30.22 & -30.47 & -35.22 & -36.47
\end{tabular}

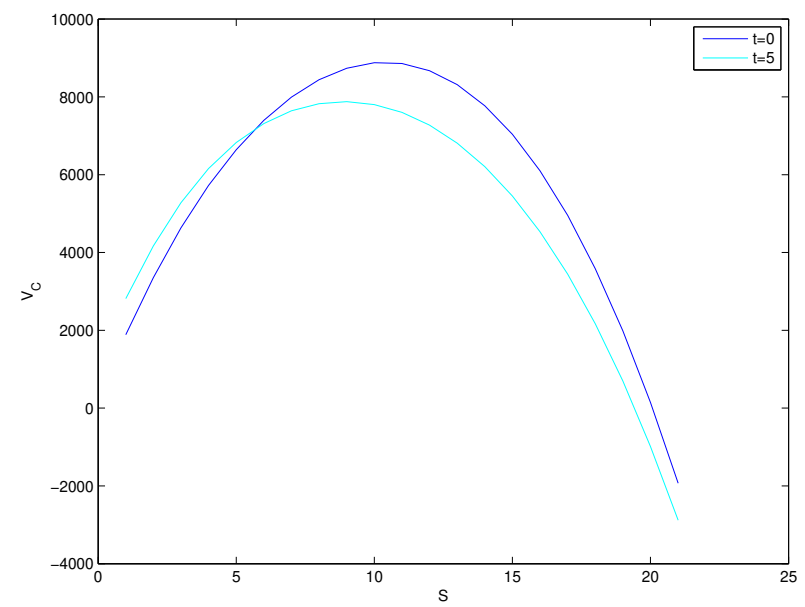

FIG. 4.4. The value functions at $t=0$ and $t=5$.

Furthermore, multi-regions transboundary air pollution problems are more common in China. In this paper, we only consider a two-region transboundary air pollution. We will consider multi-regions transboundary air pollution problems in future work. The main idea is similar to the one in this paper. Adaptive resonance theory will be used to classify the similarity of multi-region transboundary pollution problems.

Acknowledgements. The authors express their thanks to the referees for their helpful suggestions which led to improvements of the presentation.

\section{REFERENCES}

[1] H. BencheKroun AND A. ChAUdhuRI, Transboundary pollution and clean technologies, Resource Energ. Econom., 36 (2014), pp. 601-619.

[2] A. Bernard, A. Haurie, M. Vielle, And L. Viguier, A two-level dynamic game of carbon emission trading between Russia, China, and Annex B countries, J. Econom. Dyn. Contr., 32 (2008), pp. 18301856. 
[3] M. BREton, G. ZACCOUR, AND M. ZAHAF, A differential game of joint implementation of environmental projects, Automatica J. IFAC, 41 (2005), pp. 1737-1749.

[4] K. Chang, S. WANG, AND K. Peng, Mean reversion of stochastic convenience yields for $\mathrm{CO}_{2}$ emissions allowances: empirical evidence from the EU ETS, Spanish Rev. Financial Econom., 11 (2013), pp. 3945.

[5] S. CHANG, J. WANG AND X. WANG, A fitted finite volume method for real option valuation of risks in climate change, Comput. Math. Appl., 70 (2015), pp. 1198-1219.

[6] S. CHANG, X. WANG AND Z. WANG, Modeling and computation of transboundary industrial pollution with emission permits trading by stochastic differential game, Plos One, 10 (2015), Art. e0138641, 29 pages.

[7] Y. Chen And Z. Lu, High Efficient and Accuracy Numerical Methods for Optimal Control Problems, Science Press, Beijing, 2015.

[8] G. Daskalakis, D. Psychoyios, And R. Markellos, Modeling $\mathrm{CO}_{2}$ emission allowance prices and derivatives: evidence from the European trading scheme, J. Banking \& Finance, 33 (2009), pp. 12301241.

[9] N. HALL, Transboundary pollution: harmonizing international and domestic law, Univ. Michigan J. Law Reform, 40 (2007), pp. 681-746

[10] M. JENSEN AND I. SMEARS, On the convergence of finite element methods for Hamilton-Jacobi-Bellman equations, SIAM J. Numer. Anal., 51 (2013), pp. 137-162.

[11] R. LeVeque, Finite Volume Methods for Hyperbolic Problems, Cambridge University Press, Cambridge, 2004.

[12] S. LI, A differential game of transboundary industrial pollution with emission permits trading, J. Optim. Theory Appl., 163 (2014), pp. 642-659.

[13] J. List AND C. MASON, Optimal institutional arrangements for transboundary pollutants in a second-best world: evidence from a differential game with asymmetric players, J. Environ. Econom. Manag., 42 (2001), pp. 277-296.

[14] O. TAhVonen, Carbon dioxide abatement as a differential game, Europ. J. Political Econom., 10 (1994), pp. 685-705.

[15] D. YeUnG, Dynamically consistent cooperative solution in a differential game of transboundary industrial pollution, J. Optim. Theory Appl., 134 (2007), pp. 143-160.

[16] D. Yeung And L. Petrosyan, A cooperative stochastic differential game of transboundary industrial pollution, Automatica J. IFAC, 44 (2008), pp. 1532-1544.

[17] S. Youssef, Transboundary pollution, R\&D spillovers and international trade, Ann. Regional Sci., 43 (2009), pp. 235-250.

[18] S. WANG, A novel fitted finite volume method for the Black-Scholes equation governing option pricing, IMA J. Numer. Anal., 24 (2004), pp. 699-720.

[19] S. WANG, F. GAO, AND K. TEO, An upwind finite-difference method for the approximation of viscosity solutions to Hamilton-Jacobi-Bellman equations, IMA J. Math. Control Inform., 17 (2000), pp. 167-178. 